\title{
Acerca de la diatomea Didymosphenia geminata (Lyngbye) M. Schmidt: su morfología y distribución en Chile
}

\section{On the diatom Didymosphenia geminata (Lyngbye) M. Schmidt: its morphology and distribution in Chile}

\author{
Patricio Rivera ${ }^{1}$, Silvia Basualto ${ }^{2} \&$ Fabiola Cruces $^{1}$ \\ 1Departamento de Botánica, Universidad de Concepción, Casilla 160-C, Concepción, Chile \\ ${ }^{2}$ Centro EULA, Universidad de Concepción, Casilla 160-C, Concepción, Chile \\ privera@udec.cl
}

\begin{abstract}
The freshwater diatom Didymosphenia geminata was studied from Chilean material collected in Río Espolón and Río BíoBío using light and electron microscopy. Its principal morphological features are described and illustrated for the first time and comments about its relationships with other closely taxa, as well as its distribution in Chile, are given.
\end{abstract}

Didymosphenia geminata (Lyngbye) M. Schmidt, "Didymo" o "Moco de Roca" es vastamente conocida por los efectos negativos que ocasiona. Sus grandes masas de pedúnculos mucilaginosos a través de los cuales se adhiere a los sustratos rocosos de ríos, arroyos y algunas veces de lagos (Bothwell \& Kilroy 2011), pueden generar daños al turismo, alterar los habitats, modificar las comunidades de macroinvertebrados, e incluso, llegar a obstruir los filtros para captación de agua potable (Larned et al. 2007, Bergey et al. 2009).

En este trabajo informamos, por primera vez, acerca de la morfología, morfometría y variación de las características de los especímenes chilenos de $D$. geminata, y entregamos antecedentes de su distribución en el país, que en los últimos años comprende diversas Regiones del sur de Chile.

Hasta 1989, D. geminata había sido señalada en Chile para el Lago Sarmiento y para Río Cisnes (XI Región) por Asprey et al. (1964), y para Mejillones (II Región) de acuerdo con el material de Charles Boyer, slide F-4-6 rotulado Gomphonema geminatum, depositado en la Academia de Ciencias Naturales de Filadelfia, Estados Unidos (Rivera \& Gebauer 1989). En 2010, la Subsecretaría de Pesca al verificar que la especie fue encontrada en los ríos Espolón y Futaleufú, X Región (Subpesca 2010), financió estudios tendientes a clarificar su distribución en la zona sur del país. Los resultados de estos estudios (POCH 2011) indican que el taxón está presente en los ríos Risopatrón, Figueroa, Pico, Ñirehuao, Emperador Guillermo, Simpson, Aysén, D. Lago Monreal, Cochrane y Baker (XI Región), como también en la cuenca del Río Puelo, rios Yelcho, Espolón, Bellavista, Futaleufú y Noroeste (X Región). En noviembre de 2012, durante un Taller sobre Didymosphenia organizado por el Centro EULA de la Universidad de Concepción, Georgina Lembeye, del Departamento de Acuicultura de la Subsecretaría de Pesca, informó que $D$. geminata fue recientemente encontrada en el Río Llanquihue (X Región) y en la zona alta del Río Bío-Bío (IX Región).

El material estudiado está depositado en la Colección Diatomológica de la Universidad de Concepción (DIATCONC): Desembocadura del Río Espolón, Comuna de

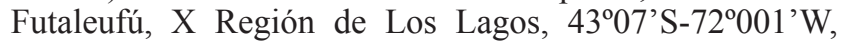
02.06.2010, DIAT-CONC M3366; Río Bío-Bío, Sector Tallón, IX Región de la Araucanía, 38 34'S-71 ${ }^{\circ} 08^{\prime} \mathrm{W}$, 21.11.2012, DIAT-CONC M3447; Río Bio-Bío, Balsa Caracoles, IX Región de la Araucanía, $38^{\circ} 20^{\prime} \mathrm{S}-71^{\circ} 17^{\prime} \mathrm{W}$, 01.12.2012, DIAT-CONC M3450.

Los ejemplares chilenos forman grandes conglomerados macroscópicos de color gris a verde marrón (Fig. 1), constituidos por pedúnculos de mucílago, de 14 a 21 $\mu \mathrm{m}$ de diámetro, secretados por el extremo basal de los frústulos, ramificados dicotómicamente (Fig. 2 A) y unidos a piedras sumergidas. En vista conectival, los frústulos tienen forma de cuña (Fig. 2 B), y hasta tres bandas abiertas, anchas, provistas de una línea de poros, fueron observadas en una semiteca (Fig. 2 C). Las valvas son de gran tamaño, 108-138 $\mu \mathrm{m}$ de largo y 33-43 $\mu \mathrm{m}$ de ancho, planas y con un bien desarrollado manto valvar, levemente asimétricas respecto al eje apical, y los extremos son claramente capitados, siendo el extremo apical de mayor tamaño que el extremo basal (Fig. 2 D-H). El rafesterno es relativamente ancho. El área central es grande, lanceolada, asimétrica en algunos ejemplares (Fig. 3 E) y lleva 2-5 estigmas a un lado del nódulo central (Fig. 3 E-G). Las aberturas externas de los estigmas son alargadas (Fig. 2 I); internamente son globosas, con aspecto cerebriforme (Fig. $3 \mathrm{H}$ ), y quedan separadas del nódulo central por una leve depresión alargada de la valva (Fig. 3 G, flecha). El rafe es central, levemente arqueado; externamente los extremos proximales son espatulados, distanciados entre sí, y terminan en una pequeña depresión algo curvada hacia los 
estigmas; las fisuras terminales se curvan bruscamente en la misma dirección, en sentido opuesto a los estigmas (Fig. 2 D-I). Internamente el rafe termina en helictoglossae bien estructuradas, inclinadas con respecto al plano apical (Fig. 3 D). Las estrías (9-10 en $10 \mu \mathrm{m})$ se extienden sobre la valva y hasta la mitad del manto valvar, dejando una ancha zona hialina que alcanza el borde de la valva (Fig. 2 C); son radiales en el centro de la valva y alternativamente largas y cortas (Fig. 3 F), luego se orientan en forma divergente hacia ambos extremos (Fig. 2 D-H), pero son radiales alrededor del extremo apical (Fig. 3 A-B). El extremo basal presenta un campo de poros formado por líneas orientadas en sentido del eje apical, 4-5 en $1 \mu \mathrm{m}$ (Fig. 3 C-D). Internamente, las interestrías son robustas y muy elevadas en toda la cara valvar (Fig. 3 F). Externamente, en la unión cara valvar /manto se encuentra un reborde silíceo que recorre la valva desde un extremo al otro (Fig. 2 I), $y$ que en ambos lados del extremo apical termina en una bien desarrollada espina (Fig. 3 A, flechas), especialmente visible en vista conectival (Fig. 2 C). Externamente las aréolas (10-11 en $10 \mu \mathrm{m})$ son de contorno cuadrangular, pentagonal o hexagonal; presentan un borde algo elevado sobre la valva, no liso, y la pared exterior de cada areola, dispuesta algo más abajo, lleva un número variable de apéndices silíceos puntiagudos (volae) que se dirigen

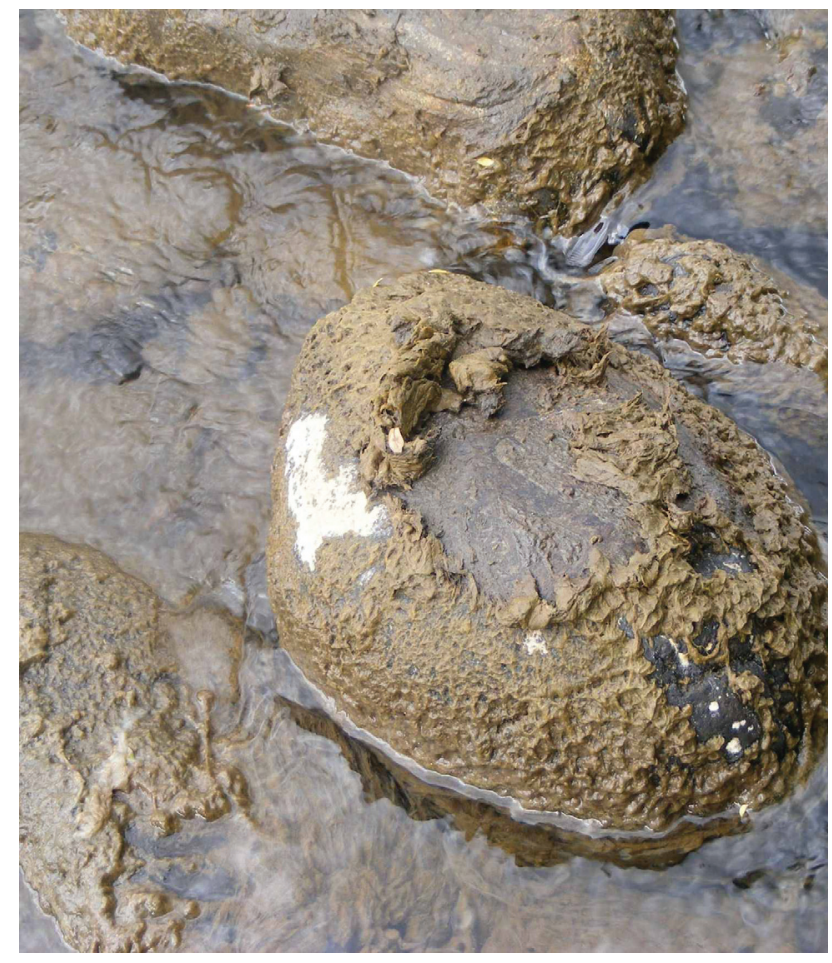

Figura 1. Conglomerados macroscópicos de D. geminata sobre piedras en Río Bío-Bío.

FiguRE 1. Macroscopic growths of D. geminata on stones in Río Bío-Bío. hacia el centro, el cual se observa de contorno subcircular (Fig. $3 \mathrm{~K}$ ). Internamente cada aréola se abre en una ranura con aspecto dendriforme (Fig. 3 I). En la cara externa de las valvas, el rafesterno y los márgenes del área central no son lisos sino provistos de un tipo de lóculos cerrados hacia el exterior, que tienen una apariencia muy similar a la de las aréolas (Fig. $3 \mathrm{~J}$ ), pero tampoco se abren hacia el interior de la célula (Fig. 3 B, D).

Didymosphenia geminata es conocida por presentar una gran variación de algunas de sus características morfológicas, principalmente el largo y ancho de las valvas, y número de estigmas (Antoine \& Benson-Evans 1983). Las características del material estudiado concuerdan con aquellas descritas por Dawson (1973) utilizando técnicas de microscopía electrónica. Las diferencias encontradas son menores y tienen que ver básicamente con la continuidad alrededor del extremo apical del reborde silíceo ubicado en el borde del manto valvar (situación nunca observada en este estudio).

Metzeltin \& Lange-Bertalot (1995) reconocen en $D$. geminata los morfotipos capitata y subcapitata. El material chileno siempre presentó el extremo apical notoriamente capitado, característica distintiva del morfotipo capitata, pero la presencia en algunos individuos de más de tres estigmas, y de 9-10 estrías en $10 \mu \mathrm{m}$ lo alejan de él. Se han descrito once especies de Didymosphenia (Whitton et al. 2009) y en tres de ellas las aréolas están hundidas en la pared valvar: Didymosphenia geminata, D. clavaherculis (Ehr.) Metzeltin \& Lange-Bertalot (1995) y D. tatrensis Mrozinska, Czerwik-Marcinkowska \& Gradzinski (2006). En D. tatrensis la morfología externa de las aréolas es completamente diferente a las dos primeras especies, como también su forma valvar, número de estrías y estigmas. $D$. clavaherculis difiere de D. geminata principalmente por sus estrías más distanciadas, 7-8 en $10 \mu \mathrm{m}$, convergentes en los extremos apical y basal, y por una mayor variación del número de estigmas (2-7).

En Chile D. geminata fue declarada plaga por la Subsecretaría de Pesca (Resolución Ex 2064/2010). Sabemos que los blooms de $D$. geminata se producen en ríos que presentan bajos niveles de nutrientes, específicamente, bajas concentraciones de fósforo (Bothwell et al. 2012). Esta característica ha llevado a que una de las medidas adoptadas para el control de la especie en los sistemas acuáticos sea el manejo de los niveles de nutrientes (Whitton et al. 2009, Bothwell \& Kilroy 2011). A nivel gubernamental, la Subsecretaría de Pesca está adoptando algunas medidas para el control de D. geminata en el país, como son la implementación de programas de monitoreo y vigilancia en diferentes regiones, de educación y entrenamiento de personal, de apoyo económico para la investigación y para la participación de los expertos en talleres y congresos (G. Lembeye, Taller D. geminata, UdeC, noviembre 2012). Por otra parte Reid et al. (2012), para prevenir la expansión del taxón en Patagonia y Sudamérica, recomiendan priorizar áreas basándose en un aislamiento geográfico y en su valor económico y de conservación. 

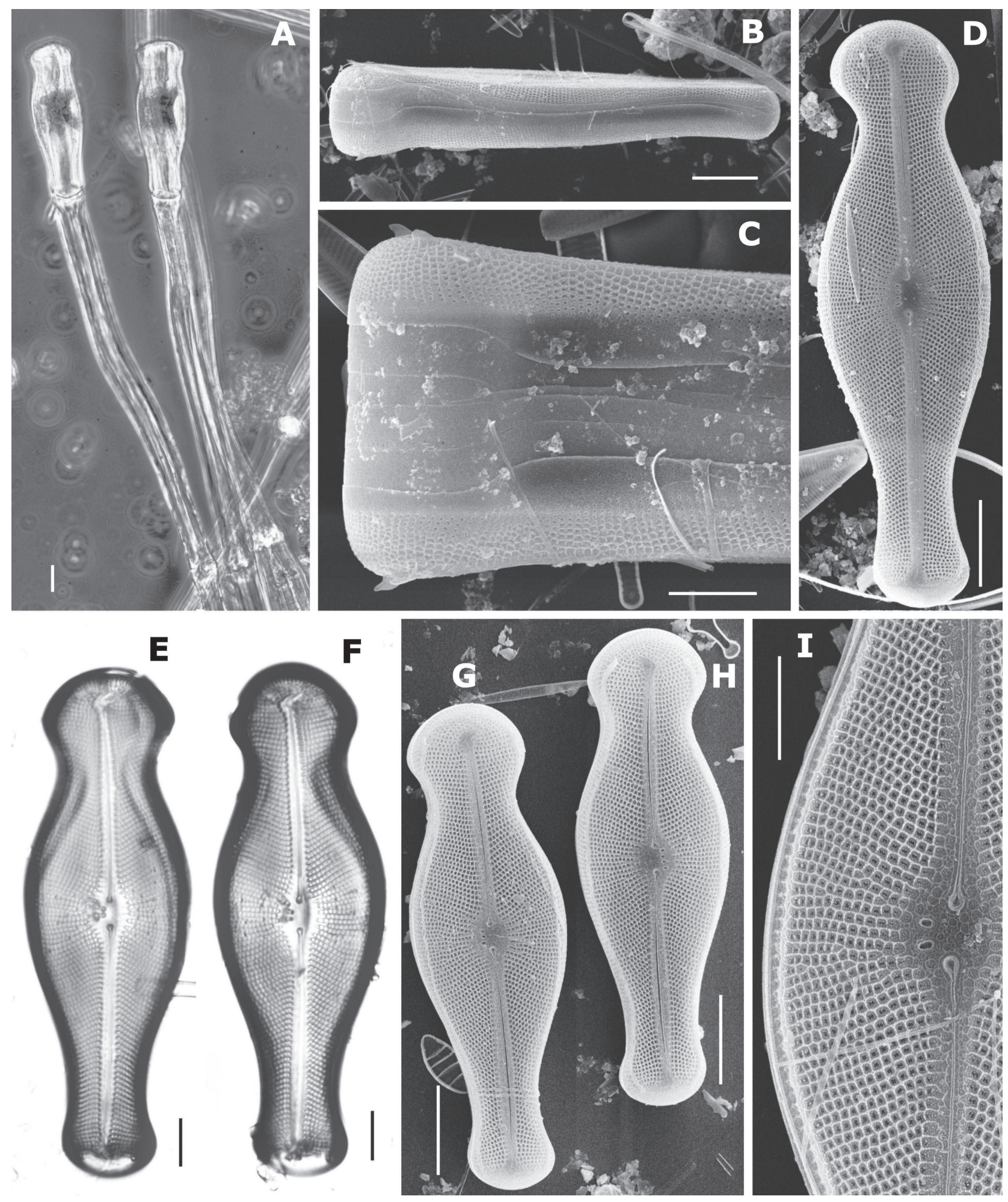

Figura 2. A. D. geminata unida a pedúnculos de mucílago. B. Frústulo en vista conectival. C. Bandas del cingulum y manto valvar. D-H. Vista valvar y orientación de las estrías. I. Area central lanceolada, dos estigmas y rafesterno ancho. Escalas: C, E-F, I = $10 \mu \mathrm{m} ; \mathrm{A}-\mathrm{B}, \mathrm{D}$, G-H $=20 \mu \mathrm{m}$. Figs. A, E-F, Microscopía Optica; Figs. B-D, G-I, Microscopía Electrónica de Barrido.

FIgURE 2. A. Stalks of D. geminata. B. Frustule in conectival view. C. Girdle bands and valvar mantle. D-H. Valve views and striae orientation. I. Lanceolate central area, two stigmata and wide raphe-sternum. Scale bars: C, E-F, I = $10 \mu \mathrm{m} ; \mathrm{A}-\mathrm{B}, \mathrm{D}, \mathrm{G}-\mathrm{H}=20 \mu \mathrm{m}$. Figs. A, E-F, Light Microscopy; Figs. B-D, G-I, Scanning Electron Microscopy. 

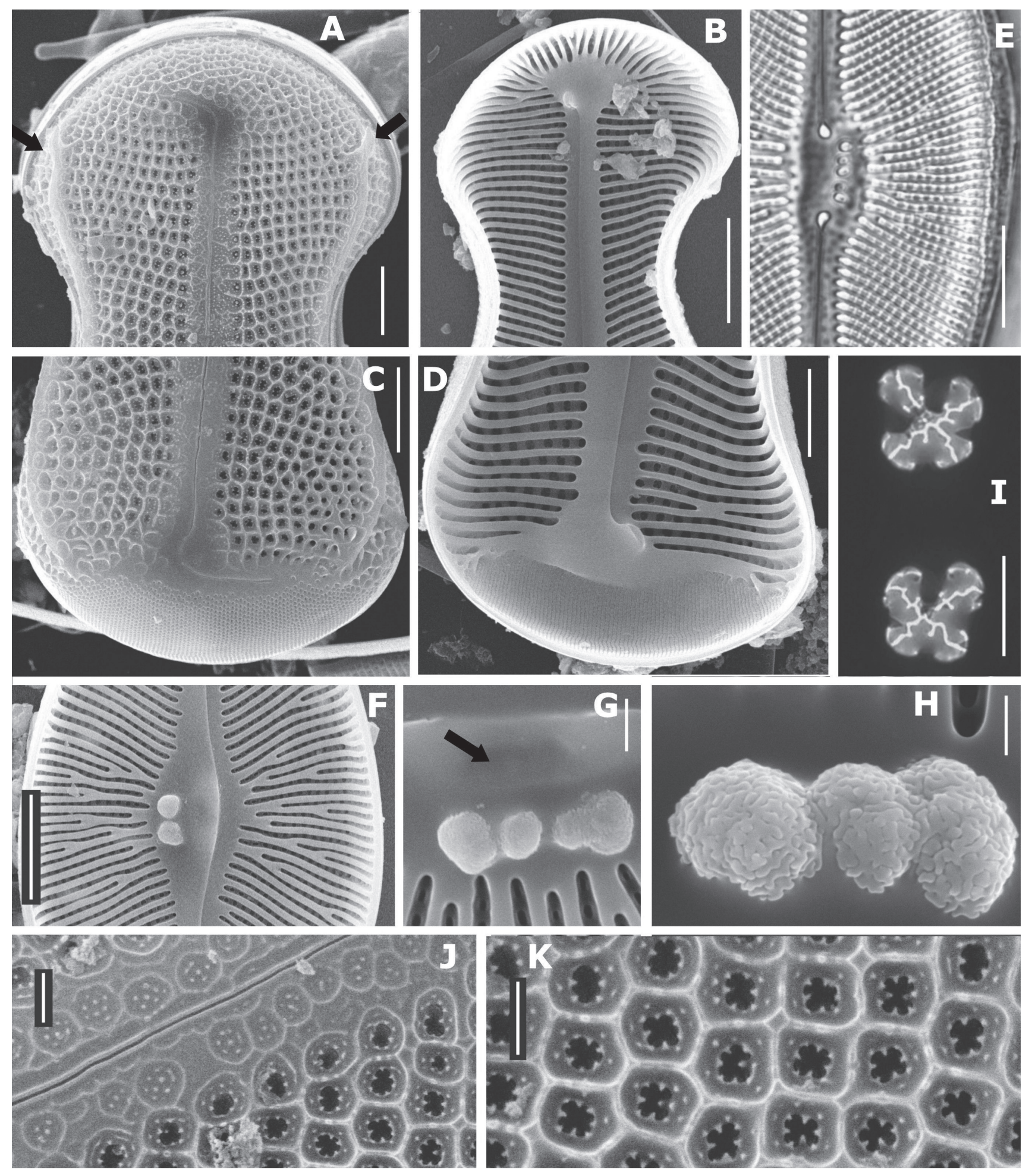

FiguRA 3. A-B. Extremo apical. C-D. Extremo basal. E-H. Variación número de estigmas. I, K. Estructura areolar. J. Rafesterno y aréolas. Escalas: A, C-D $=5 \mu \mathrm{m}$; B, E-F $=10 \mu \mathrm{m} ; \mathrm{I}=0,5 \mu \mathrm{m} ; \mathrm{G}=2 \mu \mathrm{m} ; \mathrm{H}, \mathrm{J}-\mathrm{K}=1 \mu \mathrm{m}$. Figs. A-B, C-D, F-K, Microscopía Electrónica de Barrido; Fig. E, Microscopía Optica; Fig. I, Microscopía Electrónica de Transmisión.

FIgURE 3. A-B. Apical end. C-D. Basal end. E-H. Variation in number of stigmata. I, K. Areolae structure. J. Raphe-sternum and areolae. Scale bars: A, C-D $=5 \mu \mathrm{m}$; B, E-F $=10 \mu \mathrm{m} ; \mathrm{I}=0,5 \mu \mathrm{m} ; \mathrm{G}=2 \mu \mathrm{m} ; \mathrm{H}, \mathrm{J}-\mathrm{K}=1 \mu \mathrm{m}$. Figs. A-B, C-D, F-K, Scanning Electron Microscopy; Fig. E, Light Microscopy; Fig. I, Transmission Electron Microscopy. 


\section{AGRADECIMIENTOS}

Agradecemos a la Doctora Silvia Sala y a otro revisor anónimo por sus valiosas sugerencias y comnetarios al manuscrito. Agradecemos también al personal del centro de Espectroscopía y Microscopía Electrónica de la Universidad de Concepción por su ayuda con los microscopios electrónicos. Investigación financiada parcialmente por el Proyecto FONDECYT 11100160.

\section{BIBLIOGRAFÍA}

Antorne, S.E. \& K. Benson-Evans. 1983. Polymorphism and size variation in Didymosphenia geminata from Great Britain. British Phycological Journal 18: 199-200.

Asprey, G.F., K. Benson-Evans \& J. Furet. 1964. A contribution to the study of South American freshwater phytoplankton. Gayana Botánica 10: 1-18.

Bergey, E.A., J.T. Cooper \& C.R. TACKett. 2009. Occurrence of the invasive diatom Didymosphenia geminata in Southeast Oklahoma. Publications of the Oklahoma Biological Survey, $2^{\text {nd }}$ Series, 9: 13-15.

Bothwell, M.L. \& C. Kilroy. 2011. Phosphorous limitation of the freshwater benthic diatom Didymosphenia geminata determined by the frequency of dividing cells. Freshwater Biology 56: 565-578.

Bothwell, M.L., C. Kilroy, B. Taylor, E.T. Ellison, D.A. James, C. Gillis, K.D. Bladon \& U. Silins. 2012. Iron is not responsible for Didymosphenia geminata bloom formation in phosphorous-poor rivers. Canadian Journal of Fisheries and Aquatic Sciences 69: 1723-1727.

Dawson, P. 1973. The Morphology of the Siliceous Components of Didymosphenia geminata (Lyngb.) M. Schm. British Phycological Journal 8: 65-78.
Larned, S., D. Arscott, N. Blair, B. Jarvie, D. Jellyman, K. Lister, M. Schallenberg, S. Sutherland, K. Vopel \& B. WiLcock. 2007. Ecological studies of Didymosphenia geminata in New Zealand, 2006-2007. NIWA Client Report CHC2007-070; NIWA Project MAF07507: 120 pp.

Metzeltin, D. \& H. Lange-Bertalot. 1995. Kritische Wertung der Taxa in Didymosphenia (Bacillariophyceae). Nova Hedwigia 60: 381-405.

Mrozinska, T., J. Czerwik-MarcinKowsKa \& M. GradzinSKi. 2006. A new species of Didymosphenia (Bacillariophyceae) from the Western Carpathian Mountains of Poland and Slovakia. Nova Hedwigia 83: 499-510.

Poch. 2011. Prospección de la presencia de Didymosphenia geminata en las regiones XIV, X, XI y XII y elaboración de material de difusión tendiente a su control. Para Subsecretaría de Pesca, enero 2011. 76 pp.

Reid, B., K.L. Hernández, M. Frangopulos, M. Lorca, C. Kilroy \& S. Spaulding. 2012. The invasion of the freshwater diatom Didymosphenia geminata in Patagonia: prospects, strategies, and implications for biosecurity of invasive microorganisms in continental waters. Conservation Letters 2012: 1-9.

Rivera, P. \& M. Gebauer. 1989. Diatomeas chilenas en las Colecciones de Boyer, Cleve \& Moeller, Schulze y Smith, depositadas en la Academia de Ciencias Naturales de Filadelfia, Estados Unidos. Gayana Botánica 46: 89-116.

SUBPESCA. 2010. Informe Técnico D. AC. $\mathrm{N}^{\circ}$ 2064-2010. Presencia de Didymosphenia geminata en Río Espolón y Río Futaleufú, Región de Los Lagos. Propuesta de Area de Plaga (D.S. MINECON N³45-2005). 19 pp. Anexo 4 pp.

Whitton, B.A., N.T. Ellwood \& B. Kawecka. 2009. Biology of the freshwater diatom Didymosphenia: a review. Hydrobiologia 630: 1-37.

Recibido: 07.01.13

Aceptado: 08.03.13 